\title{
A Lonely Universe
}

\author{
Charles Lineweaver
}

Andrew Snyder-Beattie, Anders Sandberg, K. Eric Drexler, and Michael Bonsall, "The Timing of Evolutionary Transitions Suggests Intelligent Life Is Rare,” Astrobiology 21, no. 3 (2021): 265-78, doi:10.1089/ast.2019.2149.

$\mathrm{I}$ N THIS PAPER, Andrew Snyder-Beattie et al. attempt to answer the question of whether intelligent life is rare in the universe. This question has often engendered a sense of circularity in which the rarity of intelligent life is justified on the grounds that it took 4.5 billion years for the question to be asked. This question has also given rise to a sense of uneasiness. Two-thirds of the known earthlike planets are older than the earth by an average of two billion years. If these planets harbor life, it has had two billion years longer to evolve. ${ }^{1}$ These facts were sufficient to persuade Stephen Hawking that we should keep our heads down. ${ }^{2}$ When it comes to clashes between civilizations, it is far better to find, than be found. ${ }^{3}$ If Snyder-Beattie et al. are correct in their conclusion that intelligent life is rare, we may not have to worry about being found by other civilizations.

In 2002, my colleague Tamara Davis and I argued that one could estimate the probability of abiogenesis on other planets based on how rapidly it occurred on earth. ${ }^{4}$ The more rapidly on earth, the more probable elsewhere. Snyder-Beattie et al. use the same reasoning to connect the rapidity of a series of evolutionary transitions on earth to the probability of analogous transitions elsewhere. To better understand this approach, consider the following example. Suppose that you are a corvid ornithologist and that you have designed a range of puzzles for crows to solve. ${ }^{5}$ Some of the puzzles are easy; some are hard; and some are near impossible-but the difficulty of each puzzle is unknown. An experiment is then designed to determine the difficulty of all the puzzles. The amount of time taken to solve each puzzle and the number of puzzles solved is tracked carefully. If this process is repeated multiple times and the crow solves many puzzles quickly, it follows that the puzzles are easy. The puzzles solved quickest are the easiest, and, therefore, crows that can solve them should be commonplace. The puzzles that take the most time to solve are the hardest, and crows that can solve them rarer.
The crow experiment is analogous to our status on earth. We have been solving evolutionary puzzles for four billion years. Current modeling suggests that the habitable lifetime of the earth will come to an end in about one billion years, mostly due to the increasing luminosity of the sun and the resultant loss of water. ${ }^{6}$ The total habitable lifetime of the earth is therefore approximately five billion years. Our current situation is akin to that of the crow 40 minutes into a 50-minute experiment. We have 10 minutes left. The major transitions in the evolution of our lineage are the puzzles we have already solved.?

In this comparison, the emergence of life on earth would have taken place near the beginning of the 50-minute experiment. We solved that puzzle quickly. From this result we can infer that the emergence of life should be common in the universe. At the 40-minute mark in the experiment, we find that our human-like intelligence evolved only about a second ago. The fact that it took so long suggests that human-like intelligence is difficult to evolve and uncommon. ${ }^{8}$ This is the basic reasoning behind Snyder-Beattie et al.'s conclusion that intelligent life in the universe is rare.


REVIOUS ANALYSES HAVE also looked at the emergence of life in conjunction with the emergence of human-like intelligence. ${ }^{9}$ Motivated by the assumption that four data points are better than two, Snyder-Beattie et al. have extended this earlier work with a Bayesian analysis of not only the timing of abiogenesis and the evolution of intelligence, but also the timing of two other major transitions: eukaryogenesis and the evolution of sexual reproduction. They conclude that intelligent life is rare in the universe because it took humans such a long time to evolve all four of the assumed prerequisites: abiogenesis, eukaryogenesis, sexual reproduction, and intelligence itself. Their Bayesian exploration of this result includes varying the timing of abiogenesis over a relatively wide range-between 4.3 and 3.5 billion years ago-and computing the effect of discovering that life emerged twice on earth..$^{10}$ They found that their conclusion no longer holds if life emerged twice; or if abiogenesis occurred earlier, say, within 10 million years of habitability; or if the habitable lifetime of the earth is 10 times longer than expected. ${ }^{11}$ 
Recent exoplanet studies strongly suggest that every star has some kind of planetary system and that earth-like planets are likely common in such systems. ${ }^{12}$ The earth may well be representative of a very large group of wet, rocky planets. But what about atmospheric composition, ocean volume, plate tectonics, spin period, orbital period, obliquity, the presence of a large moon, and the timing of large impacts? If the emergence and evolution of life are dependent on some of these additional details, the number of earth-like planets could be quite small. ${ }^{13}$

Once life has emerged from prebiotic chemistry, the strongest selection pressures on the evolution of a species come from other life forms: conspecifics, parasites, predators, diseases, viruses, and ecosystem variability. This self-referential nature of biology makes evolution a historical science characterized by the quirks of contingency. This characterization of evolution remains controversial. ${ }^{14}$ Our ability to extrapolate crow-puzzle experiments to crows on other planets depends on the existence of extraterrestrial crows. Similarly, the Snyder-Beattie et al. result depends on the assumption that "intelligent life elsewhere requires analogous evolutionary transitions." The validity of the Snyder-Beattie et al. result, among others, ${ }^{15}$ is dependent on the assumption that the major transitions that characterize our evolution happen elsewhere. ${ }^{16}$

There is little evidence in the history of life on earth to support this assumption. Although abiogenesis is a transition shared by the lineages of all known life on earth, diverging lineages over the next four billion years are punctuated by their own evolutionary transitions. After diverging from other life forms, transitions within our own eukaryotic lineage include eukaryogenesis, sexual reproduction, and intelligence. A general feature of these transitions in the tree of life is that the closer a transition is to the end of a branch, the more recent, specific, and uncommon it is. ${ }^{17}$ In our lineage, eukaryogenesis occurred about two billion years ago and the transition to sexual reproduction about a billion years ago. The transition to intelligence is much more recent and its timing depends on how intelligence is defined. The transition to humanlike intelligence or technological intelligence occurred only about 100,000 years ago and is species-specific. The latter trait is strong evidence we should not expect to find it elsewhere. ${ }^{18}$

Naturally, we consider the transitions that happened along our lineage to be the most important in the tree of life. But this is not a reasonable basis for assuming that our transitions are common elsewhere. Our sense of self-importance provides no objective reason to model evolution as a progression toward Homo sapiens. ${ }^{19}$ Such modeling is what Snyder-Beattie et al. and many other scientists have done.

I S IT REASONABLE to argue that among the features of life on earth, the most likely to appear in life elsewhere are those that have evolved independently many times, such as complex multicellularity, eyes, wings, and canines? ${ }^{20}$ Not really. These examples of convergent evolution have only occurred within a unique eukaryotic branch that represents a tiny fraction of the diversity of life on earth. The absence of these features elsewhere in the tree of life suggests that eukaryote evolution brought with it fundamental and deeply homologous adaptations necessary for the emergence of these features. If eukaryogenesis were a convergent feature of evolution, we would expect it to have evolved on multiple occasions among the hundreds of independent major lineages. It did not. As a result, we have no evidence that eukaryogenesis is likely to appear outside our own tree of life. The same can be said for the two other major transitions that occurred during the evolution of our lineage. These transitions did not occur in any of the hundreds of non-eukaryotic lineages. Eukaryogenesis, sexual reproduction, ${ }^{21}$ and human-like intelligence evolved only in our own lineage. ${ }^{22}$

\section{Charles Lineweaver is an Associate Professor at the Research School of Astronomy and Astrophysics at the Australian National University.}

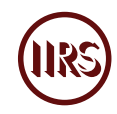

1. Charles Lineweaver, "An Estimate of the Age Distribution of Terrestrial Planets in the Universe: Quantifying Metallicity as a Selection Effect," Icarus 151, no. 2 (2001): 307-13, doi:10.1006/icar.2001.6607.

2. Paul Rincon, "Stephen Hawking's Warnings: What He Predicted for the Future," BBC News, March 15, 2018.

3. Jared Diamond, Guns, Germs and Steel: A Short History of Everybody for the Last 13,000 Years (London: Jonathan Cape, 1997).

4. Charles Lineweaver and Tamara Davis, "Does the Rapid Appearance of Life on Earth Suggest that Life Is Common in the Universe?," Astrobiology 2, no. 3 (2004): 293-304, doi:10.1089/153110702762027871.

5. Such crow experiments can be seen here: "Crow Solves an 8 Step Puzzle to Get Food. Incredible!,” YouTube video, uploaded April 3, 2014; "Causal Understanding of Water Displacement by a Crow," YouTube video, uploaded March 26, 2014; "Are Crows the Ultimate Problem Solvers? | Inside the Animal Mind | BBC Earth," YouTube video, uploaded September 10, 2014; and "Can Wild Parrots Solve Puzzles?," YouTube video, uploaded June 2, 2020.

6. Ken Caldeira and James Kasting, "The Life Span of the Biosphere Revisited," Nature 360, no. 6,406 (1992): 721-23, doi:10.1038/360721a0; and Siegfried Franck, Christine Bounama, and Werner von Bloh, "Causes and Timing of Future Biosphere Extinctions," Biogeosciences 3, no. 1: (2006) 85-92, doi:10.5194/bg-3-85-2006.

7. John Maynard Smith and Eörs Szathmáry, The Major Transitions in Evolution (Oxford, UK: Oxford University Press, 1997). 
8. If we had found that human-like intelligence had evolved much closer to the end of the habitable lifetime of the Earth we would conclude that the evolution of human-like intelligence is even rarer. The longer it has taken us (within the time allotted) to evolve something, the rarer that something should be in the universe.

9. Brandon Carter, "The Anthropic Principle and Its Implications for Biological Evolution," Philosophical Transactions of the Royal Society of London A: Mathematical, Physical and Engineering Sciences 310, no. 1,512 (1983): 347-63, doi:10.1098/rsta.1983.0096; Robin Hanson, "The Great Filter-Are We Almost Past It?" (1998); Robin Hanson, “Must Early Life Be Easy? The Rhythm of Major Evolutionary Transitions" (1998); Lineweaver and Davis, "Does the Rapid Appearance of Life on Earth Suggest that Life Is Common in the Universe?"; Charles Lineweaver and Tamara Davis, "On the Nonobservability of Recent Biogenesis," Astrobiology 3, no. 2 (2003): 241-43, doi:10.1089/153110703769016316; Victor Flambaum, "Comment on 'Does the Rapid Appearance of Life on Earth Suggest that Life Is Common in the Universe?" Astrobiology 3, no. 2 (2003): 237-39, doi:10.1089/153110703769016307; David Spiegel and Edwin Turner, "Bayesian Analysis of the Astrobiological Implications of Life's Early Emergence on Earth,” Proceedings of the National Academy of Science 109, no. 2 (2012): 395-400, doi:10.1073/pnas.1111694108; and Jingjing Chen and David Kipping, "On the Rate of Abiogenesis from a Bayesian Informatics Perspective,” Astrobiology 18, no. 12 (2018): 1,574-84, doi:10.1089/ast.2018.1836.

10. Paul Davies and Charles Lineweaver, "Finding a Second Sample of Life on Earth,” Astrobiology 5, no. 2 (2005): 15463, doi:10.1089/ast.2005.5.154.

11. See the top panel of Figure 2 in Andrew Snyder-Beattie et al., "The Timing of Evolutionary Transitions Suggests Intelligent Life Is Rare,” Astrobiology 21, no. 3 (2021): 265-78, doi:10.1089/ast.2019.2149.

12. Charles Lineweaver and Aditya Chopra, "The Habitability of Our Earth and Other Earths: Astrophysical, Geochemical, Geophysical, and Biological Limits on Planet Habitability," Annual Review of Earth and Planetary Sciences 40, no. 1 (2012), doi:10.1146/annurev-earth-042711-105531; Josh Winn and Daniel Fabrycky, "The Occurrence and Architecture of Exoplanetary Systems," Annual Review of Astronomy and Astrophysics 53, no. 1 (2015), doi:10.1146/annurev-astro-082214-122246; Danley Hsu et al., "Occurrence Rates of Planets Orbiting FGK Stars: Combining Kepler DR25, Gaia DR2, and Bayesian Inference," Astronomical Journal 158, no. 3 (2019): 109, doi:10.3847/1538-3881/ab31ab; and Steve Bryson et al., "The Occurrence of Rocky Habitable-Zone Planets around Solar-Like Stars from Kepler Data," Astronomical Journal 161 (2021): 36, doi:10.3847/1538-3881/abc418.

13. Peter Ward and Donald Brownlee, Rare Earth (New York: Copernicus, 2000); and David Waltham, Lucky Planet (New York: Basic Books, 2014)

14. In agreement with my view are George Simpson, Stephen Jay Gould, and Ernst Mayr. Simpson writes:
Progress leading to man was inherent in the evolutionary process only in the banal sense that it did occur, and progress leading to, say, an orchid or a flea was no less inherent in the same sense.

Similarly, Gould once remarked:

Humans arose, rather, as a fortuitous and contingent outcome of thousands of linked events, any one of which could have occurred differently and sent history on an alternative pathway that would not have led to consciousness.

See George Simpson, The Meaning of Evolution (New Haven: Yale University Press, 1967), 262; Stephen Jay Gould, “The Evolution of Life on the Earth," Scientific American 271, no. 4 (1994): 85-91, doi:10.1038/scientificamerican1094-84; and Ernst Mayr, What Is Evolution? (London: Weidenfeld \& Nicolson, 2001), 212-13.

Dissenting views can be found in Simon Conway Morris, Life's Solution: Inevitable Humans in a Lonely Universe (Cambridge, UK: Cambridge University Press, 2003); Simon Conway Morris, "Predicting What Extra-Terrestrials Will Be Like: And Preparing for the Worst," Philosophical Transactions of the Royal Society A: Mathematical, Physical, and Engineering Sciences 369, no. 1,936 (2011): 555-71, doi:10.1098/rsta.2010.0276; and William Bains and Dirk Schulze-Makuch, “The Cosmic Zoo: The (Near) Inevitability of the Evolution of Complex, Macroscopic Life," Life 6, no. 3 (2016): 25, doi:10:3390/life6030025.

15. Carter, "The Anthropic Principle and Its Implications for Biological Evolution"; Hanson, "The Great Filter"; Hanson, “Must Early Life Be Easy?”; Flambaum, "Comment on 'Does the Rapid Appearance of Life on Earth Suggest that Life Is Common in the Universe?'”; Brandon Carter, "Five- or Six-Step Scenario for Evolution?" International Journal of Astrobiology 7, no. 2 (2008): 177-82, doi:10.1017/ s1473550408004023; and Bains and Schulze-Makuch, "The Cosmic Zoo.”

16. In trying to assess the significance of this paper, it is helpful to keep in mind the important difference between "did" and "does." "How long did it take?" (for us in the past during the evolution of our lineage) is extrapolated to answer the question "How long does it take?" (on average during the generic evolution of other life forms). One important limitation: only if something like life-as-we-know-it exists elsewhere, could the "did" of the evolution of our lineage have a chance of being representative of the "does" of this other life.

17. For a visual representation, see Figure 1 in Cindy Castelle and Jillian Banfield, "Major New Microbial Groups Expand Diversity and Alter Our Understanding of the Tree of Life," Cell 172, no. 6 (2018): 1,181-97, doi:10.1016/j.cell.2018.02.016.

18. Gaylord Simpson, "The Nonprevalence of Humanoids," Science 143, no. 3,608 (1964): 769-75, doi:10.1126/science.143.3608.769; Charles Lineweaver, "Book Review: Intelligent Life in the Universe: From Common Origins to the 
Future of Humanity," Astrobiology 5, no. 5 (2005): 658-61, doi:10.1089/ast.2005.5.658; Charles Lineweaver, "Paleontological Tests: Human-Like Intelligence Is Not a Convergent Feature of Evolution," in From Fossils to Astrobiology, ed. Joseph Seckbach and Maud Walsh (Dordrecht: Springer, 2009), 353-68; Ernst Mayr, "Can SETI Succeed? Not Likely," Bioastronomy News 7, no. 3 (1995); and Carl Sagan, "The Abundance of Life-Bearing Planets," Bioastronomy News 7, no. 4 (1995).

19. Stephen Jay Gould, Wonderful Life: The Burgess Shale and the Nature of History (New York: W. W. Norton \& Company, 1990); Stephen Jay Gould, The Structure of Evolutionary Theory (Cambridge, MA: Harvard University Press, 2002); Gould, "The Evolution of Life on the Earth"; Mayr, What Is Evolution?, 212-13; Simpson, The Meaning of Evolution; and Stuart Kauffman, "Evolution beyond Newton, Darwin and Entailing Law," in Complexity and the Arrow of Time, ed. Charles Lineweaver, Paul Davies, and Michael Ruse (Cambridge, UK: Cambridge University Press, 2013), 162-90, doi:10.1017/CBO9781139225700.011.

20. On multicellularity particularly, see Richard Grosberg and Richard Strathmann, "The Evolution of Multicellularity: A Minor Major Transition," Annual Review of Ecology, Evolution, and Systematics 38 (2007): 621-54, doi:10.1146/annurev. ecolsys.36.102403.114735; and Andrew Knoll, "The Multiple Origins of Complex Multicellularity," Annual Review of Earth and Planetary Science 39 (2011): 217-39, doi:10.1146/ annurev.earth.031208.100209. More generally, see Morris, Life's Solution; Morris, "Predicting What Extra-Terrestrials Will Be Like"; Simon Conway Morris, Runes of Evolution: How the Universe Became Self-Aware (West Conshohocken: Templeton Press, 2015); and George McGhee Jr., Convergent Evolution on Earth: Lessons for the Search for Extraterrestrial Life (Cambridge, MA: MIT Press, 2019).

21. I am referring here to eukaryotic sexual reproduction involving meiosis, not to bacterial transduction, conjugation, or transformation, which are sometimes called "bacterial sex."

22. Simpson, "The Nonprevalence of Humanoids"; Lineweaver, "Book Review: Intelligent Life in the Universe"; Lineweaver, "Paleontological Tests"; Mayr, "Can SETI Succeed? Not Likely"; and Sagan, "The Abundance of Life-Bearing Planets."

DOI: $10.37282 / 991819.22 .11$

Published on February 2, 2022

https://inference-review.com/article/a-lonely-universe 